\title{
A CONVERSE OF TAYLOR'S THEOREM FOR FUNCTIONS ON BANACH SPACES
}

\author{
S. DAYAL
}

\begin{abstract}
The main results are the local representation theorems associating the local weak $n$-Taylor series expansion of a function defined on a Banach space to a local $n$-Taylor series expansion of the coefficients. These theorems are used to prove a converse of Taylor's theorem which uses weaker hyptohesis than used by others. Another useful application of the above results is done in [2] to study a class of functions called $n$-convex functions.
\end{abstract}

1. Introduction. The first main result (Theorem 3.2) states that if a function $f$ defined on a Banach space has a weak $n$-Taylor series expansion (see definition in \$2) throughout an open set and if the $n$th order coefficient is continuous at one point, then the coefficients have strong Taylor series expansion (see definition in \$2) about every point near to that point. The proof of this theorem uses a sort of mean value theorem (Theorem 3.1). A particular case of Theorem 3.1 is proved by McLeod [11] and Dieudonné [5]. Theorem 3.3 generalizes Theorem 3.2 in the sense that the $n$th order coefficient has a strong $m$-Taylor series expansion about a point instead of being continuous at that point. A useful application of Theorem 3.2 is done by the author [2] to study the question of existence of higher differentials of $n$-convex functions which contain a class of functions called subconvex functions.

The second main result (Theorem 4.1) states that under the situation of Theorem 3.2 if the coefficients are bounded then the coefficients of the weak Taylor series are in fact iterated Fréchet differentials. This is a converse of Taylor's theorem which uses weaker hypothesis than used by Nashed [12] and Abraham and Robin [1]. In fact it does not use the superfluous condition of continuity of lower order coefficients which is used by Abraham and Robin.

2. Preliminaries. In this section we first give the meanings of differentiability of functions on Banach spaces, weak and strong Taylor series expansion and the notion of discrete difference together with its properties.

Let $E$ and $F$ be two Banach spaces and $A$ be an open set in $E$. A function

Received by the editors January 7, 1976 and, in revised form, November 9, 1976.

AMS (MOS) subject classifications (1970). Primary 41A65, 58C20.

Key words and phrases. Fréchet differential, strong differential, multilinear functions, discrete differences, convex property, convex set, local representations, weak and strong $n$-Taylor series expansion. 
$f: A \rightarrow F$ is said to be Fréchet differentiable at $x \in A$, if there is a bounded linear transformation $\lambda_{x} \in L(E, F)$ the space of bounded linear transformation on $E$ to $F$ with the property that for all $\varepsilon>0$ there is a $\delta>0$ such that $|t|<\delta$ implies $\left\|f(x+t u)-f x-\lambda_{x}(t u)\right\|<\varepsilon \cdot|t|$ uniformly with respect to $u \in E\|u\|<1$. If $f$ is Fréchet differentiable at every point $x \in A$, it determines a vector valued function $f^{\prime}: A \rightarrow L(E, F)$ by the formula $f^{\prime} x=\lambda_{x}$. Thus if $f$ is Fréchet differentiable at $x$ the Fréchet differential of $f$ at $x$ can be denoted by $f^{\prime} x$. The Fréchet differentiation process can be iterated. If $f$ : $A \rightarrow F$ is Fréchet differentiable in $A$, the vector valued function $f^{\prime}: A \rightarrow$ $L(E, F)$ may again be Fréchet differentiable in the above sense, thus we may have again a vector valued function $f^{\prime \prime}: A \rightarrow L(E, L(E, F))$ and in general $f^{(n)}: A \rightarrow L(E, L(E, \ldots, L(E, F)))$ which define the higher Fréchet differential. Because of natural isometry we may regard $L(E, L(E, F))$ as equivalent to $L_{2}(E, F)$ the space of bounded bilinear transformations from $E^{2} \rightarrow F$ and in general $f^{(n)}: A \rightarrow L_{n}(E, F), L_{n}(E, F)$ the space of bounded multilinear transformation from $E^{n} \rightarrow F$. In fact the correspondence is even simpler to translate for Fréchet differential because $f^{(n)} x$ is symmetric [5, p. 178]. Some properties of Fréchet differential may be found in [5]. $f: A \rightarrow F$ is said to have strong differential at $x$ if there is a bounded linear transformation $f^{\prime} x \in$ $L(E, F)$ with the property that $f x_{1}-f x_{0}=\left(f^{\prime} x\right)\left(x_{1}-x_{0}\right)+\left(x_{1}-x_{0}\right)$. $r\left(x_{1}, x_{0}\right)$, where $\lim _{\left(x_{1}, x_{0}\right) \rightarrow(x, x)} r\left(x_{1}, x_{0}\right) \rightarrow 0$. The strong differential is defined and used in [9].

We say $f: A \rightarrow F$ has a weak $n$-Taylor series expansion about $y \in A$ if there are symmetric $k$-linear functions $P_{k}(y)$, possibly unbounded, such that

$$
f(y+t u)=\sum_{k=0}^{n} \frac{1}{k !} \cdot P_{k}(y)(t u)^{k}+r(u, t)
$$

where $\lim _{t \rightarrow 0} r(u, t) / t^{n}=0$.

If the limit is uniform for vectors $u,\|u\| \leqslant 1$, then (2.1) is a strong $n$-Taylor series expansion about $y$.

Let $g_{0}: E \rightarrow F$ be constant valued function and $g_{k}: E^{k} \rightarrow F$ be $k$-linear (possibly unbounded) operators for $1 \leqslant k \leqslant n$, where $E^{k}$ is the $k$-fold cartesian product of $E$. The operator $P: E \rightarrow F$ given by

$$
P(x)=\hat{g}_{0}(x)+\hat{g}_{1}(x)+\cdots+\hat{g}_{n}(x),
$$

where $\hat{g}_{k}(x)=g_{k}(x, x, \ldots, x)$ is induced by $g_{k}$, is called a polynomial of degree $<n$. In case of $E=R$, let $y_{k}=g_{k}(1)$ and then put $p(t)=\sum_{k=0}^{n} t^{k} \cdot y_{k}$. Let $f:[a, b] \rightarrow F$ and $t=\left(t_{0}, t_{1}, \ldots, t_{n}\right)$ be a sequence of distinct numbers from $[a, b]$. A discrete difference $\left[\Delta_{n} f\right]\left(t_{0}, t_{1}, \ldots, t_{n}\right)$ is the coefficient of $t^{n}$ in the unique polynomial of degree $\leqslant n$ such that $P\left(t_{i}\right)=f\left(t_{i}\right)$ for $i=$ $0,1, \ldots, n$. Thus

$$
\left[\Delta_{n} f\right]\left(t_{0}, t_{1}, \ldots, t_{n}\right)=\sum_{k=0}^{n}\left(1 /\left(j \in \prod_{\substack{j \in[0, n] \\ j \neq k}}\left(t_{k}-t_{j}\right)\right)\right) \cdot f\left(t_{k}\right) .
$$


Discrete differences are evidently the extension to vector valued functions of divided differences of numerical analysis [7].

The discrete differences associated with $f:[a, b] \rightarrow F$ satisfy a convex property: namely, for $t_{0}<t<t_{n}$ with $t_{i} \neq t_{j}$ for $i \neq j$ and $t \neq t_{i}, i=$ $1,2,3, \ldots, n$,

$$
\begin{aligned}
{\left[\Delta_{n} f\right]\left(t_{0}, t_{1}, \ldots, t_{n}\right)=} & \frac{t-t_{0}}{t_{n}-t_{0}}\left[\Delta_{n} f\right]\left(t_{0}, \ldots, t_{n-1}, t\right) \\
& +\left(1-\frac{t-t_{0}}{t_{n}-t_{0}}\right) \cdot\left[\Delta_{n} f\right]\left(t_{1}, \ldots, t_{n}, t\right) .
\end{aligned}
$$

This can easily be proved by using the fact that $P_{1}$ and $P_{2}$ are polymonials of degree $<n$ such that $P_{1}$ coincides $f$ at $t_{0}, t_{1}, \ldots, t_{n}$ and $P_{2}$ coincides $f$ at $t_{1}, t_{2}, \ldots, t_{n}, t$. Then there is a polynomial given by

$$
P(s)=\frac{\left(s-t_{0}\right)}{\left(t-t_{0}\right)} \cdot P_{2}(s)+\frac{(s-t)}{\left(t_{0}-t\right)} \cdot P_{1}(s),
$$

which coincides with $f$ at $t_{0}, t_{1}, t_{2}, \ldots, t_{n}, t$. Consequently

$$
\left[\Delta_{n+1} f\right]\left(t_{0}, \ldots, t_{n}, t\right)=\frac{\left[\Delta_{n} f\right]\left(t_{0}, \ldots, t_{n-1}, t\right)-\left[\Delta_{n} f\right]\left(t_{0}, \ldots, t_{n}\right)}{t-t_{n}}
$$

and similarly

$$
\begin{aligned}
& {\left[\Delta_{n+1} f\right]\left(t_{0}, t_{1}, \ldots, t_{n}, t\right)} \\
& \quad=\frac{\left[\Delta_{n} f\right]\left(t_{1}, t_{2}, \ldots, t_{n}, t\right)-\left[\Delta_{n} f\right]\left(t_{0}, \ldots, t_{n-1}, t_{n}\right)}{t-t_{0}} .
\end{aligned}
$$

The expressions (2.5) and (2.6) give (2.4).

Let $\alpha: E^{n} \rightarrow F$ be an $n$-linear symmetric operator. If $\|\alpha\|=$ $\sup _{\left\|u_{\|}\right\|<1}\left\|\alpha\left(u_{1}, \ldots, u_{n}\right)\right\|$,

$$
\sup _{\|u\|<1}\|\alpha(u, u, \ldots, u)\| \leqslant\|\alpha\| \leqslant\left(n^{n} / n !\right) \cdot \sup _{\|u\|<1}\|\alpha(u, u, \ldots, u)\| .
$$

This inequality was first proved by Taylor [13], and Kopeć and Musiclak [8] proved that is is the best possible inequality.

3. Local representation theorems. In this section we will prove a sort of mean value theorem before we prove the main theorems.

ThroREM 3.1. Let $f: A \rightarrow F$ and $f$ have a weak $n$-Taylor series expansion about every point of some neighbourhood $N$ of a point $x \in A$. If $y$ and $u$ are vectors such that the line segment $\{y+t u: 0 \leq t \leq 1\}$ is in $N$, then the vector $f(y+u)-\sum_{k=0}^{n-1}\left(P_{k}(y)\right) u^{k} / k !$ is in the closure of the convex set $C$ spanned by the vectors $\left\{(1 / n !) \cdot\left(P_{n}(y+t u)\right) u^{n}: 0 \leqslant t \leqslant 1\right\}$.

Proof. Let $h(t)=f(y+t u)-\sum_{k=0}^{n-1}\left(t^{k} / k !\right)\left(P_{k}(y)\right) u^{k}$, so that we are concerned with proving that $h(1)$ is in the closure of $C$. We note by the 
hypothesis of weak $n$-Taylor series expansion

$$
\lim _{t \rightarrow 0} h(t) / t^{n-1}=0 .
$$

For any positive number $\delta<1 / n$, let $Q_{\delta}$ be the polynomial of degree $\leqslant n-1$ with coefficients in $F$ that coincides with $h$ at the points $0, \delta, 2$. $\delta, \ldots,(n-1) \cdot \delta$. Using Lagrange's formula [7],

$$
Q(t)=\sum_{k=0}^{n-1}\left[\left(\prod_{\substack{j \in[0, n-1] \\ j \neq k}}(t-j \cdot \delta)\right) /\left[\prod_{\substack{j \in[0, n-1] \\ j \neq k}}(k-j) \cdot \delta\right)\right] \cdot h(k \cdot \delta)
$$

(3.1) and (3.2) show that $\lim _{\delta \rightarrow 0} Q_{\delta}(t)=0$, for all $t$, and so $\lim _{\delta \rightarrow 0} Q_{\delta}(1)=0$, $h(1)=\lim _{\delta \rightarrow 0}\left(h(1)-Q_{\delta}(1)\right)$.

The $n$th degree polynomial which coincides with $h$ at $0, \delta, \ldots,(n-1) \cdot \delta$, 1 is given by

$$
Q_{\delta}(t)+\left[\Delta_{n} h\right](0, \delta, \ldots,(n-1) \cdot \delta, t) \cdot\left(\prod_{k=0}^{n-1}(t-k \cdot \delta)\right)
$$

Then

$$
\begin{aligned}
h(1) & =\lim _{\delta \rightarrow 0} Q_{\delta}(1)+\lim _{\delta \rightarrow 0}\left[\Delta_{n} h\right](0, \delta, \ldots,(n-1) \cdot \delta, 1) \cdot\left(\prod_{k=0}^{n-1}(1-k \cdot \delta)\right) \\
& =\lim _{\delta \rightarrow 0}\left[\Delta_{n} h\right](0, \delta, \ldots,(n-1) \cdot \delta, 1) .
\end{aligned}
$$

It is sufficient to prove that $\left[\Delta_{n} h\right]\left(t_{0}, t_{1}, \ldots, t_{n}\right)$ is in the closure of $C$, provided $0 \leqslant t_{0}<t_{1}<\cdots<t_{n} \leqslant 1$ and each $t_{k}$ is rational. Suppose $t_{0}<\tilde{t}$ $<t_{n}$ and $\tilde{t}$ is different from any of the $t_{k}$, then by using a similar expression as (2.4) the convex property of the discrete differences, $\left[\Delta_{n} h\right]\left(t_{0}, \ldots, t_{n}\right)$, is a convex combination of $\left[\Delta_{n} h\right]\left(\tilde{t}, t_{1}, \ldots, t_{n}\right)$ and $\left[\Delta_{n} h\right]\left(t_{0}, \ldots, t_{n-1}, \tilde{t}\right)$. If $\left[\Delta_{n} h\right]\left(t_{0}, t_{1}, \ldots, t_{n}\right)$ has a distance greater than $\varepsilon>0$ from the convex set $C$, then one of the discrete differences $\left[\Delta_{n} h\right]\left(\tilde{t}, t_{1}, \ldots, t_{n}\right)$ and $\left[\Delta_{n} h\right]\left(t_{0}, \ldots, t_{n-1}, \tilde{t}\right)$ is farther than $\varepsilon$ from $C$.

Suppose the rational numbers $t_{k}$ are of the form $p_{k} / q$. If they are not equally spaced, the integers $p_{k}$ have gaps. If $\tilde{t}=\tilde{p} / q$ is chosen to fill one of the gaps, then each of the sequences $\left(\tilde{t}, t_{1}, t_{2}, \ldots, t_{n}\right)$ and $\left(t_{0}, t_{1}, \ldots, t_{n-1}, \tilde{t}\right)$ (properly ordered) has at least one less gap and we may substitute one of these sequences for $t$. This process may be repeated until we come to the sequence $t^{1}=\left(t_{0}^{1}, \ldots, t_{n}^{1}\right)$ with $t_{k+1}^{1}-t_{k}^{1}=1 / q$ such that $\left[\Delta_{n} h\right] t^{1}$ is farther than $\varepsilon$ from $C$. The process may be repeated with $q$ replaced by $2 \cdot q, 2^{2}$. $q, \ldots$, and we obtain a sequence $t=\left\{t^{m}\right\}$ of finite sequences such that for each $m,\left[\Delta_{n} h\right] t^{m}$ is farther than $\varepsilon$ from $C . t_{k+1}^{m}-t_{k}^{m}=1 / 2^{m-1} \cdot q, t_{0}^{m}$ increases and $t_{n}^{m}$ decreases as $m$ increases, both approaching to the same limit $\bar{t}$. Now $h$ has the same $n$th discrete difference as $\bar{h}$ where $\bar{h}(t)=f(y+t u)$, since the $n$th order discrete difference of a polynomial of degree $\leqslant n-1$ are 
0. By the weak Taylor series expansion of $f$ at $y+\overline{t u}$,

$$
\bar{h}(t)=\sum_{k=0}^{n}\left((t-\bar{t})^{k} / k !\right) \cdot\left(P_{k}(y+\bar{t} u)\right) u^{k}+\bar{r}(t, u),
$$

and $\bar{r}(t, u) /(t-\bar{t})^{n} \rightarrow 0$ as $t \rightarrow \bar{t}$. Then $\lim _{m \rightarrow \infty}\left[\Delta_{n} \bar{r}\right] t^{m}=0$, because of Lagrange's formula [7], the equal spacing of $t^{m}$ and the order of magnitude of $\bar{r}$. Then

$$
\lim _{m \rightarrow \infty}\left[\Delta_{n} h\right] t^{m}=\lim _{m \rightarrow \infty}\left[\Delta_{n} \bar{h}\right] t^{m}=\frac{\left(P_{n}(y+\bar{t} u)\right) u^{n}}{n !} \in C .
$$

This contradicts the construction of the $t^{m}$ to satisfy that $\left[\Delta_{n} h\right] t^{m}$ is farther than $\varepsilon$ from $C$ for each $m$.

COROllary 3.1. Let $f: A \rightarrow F$ and $f$ have a weak $n$-Taylor series expansion about every point of some neighbourhood $N$ of a point $x \in A$. If $y$ and $u$ are vectors such that the line segment $\{y+t u: 0 \leqslant t \leqslant 1\}$ is in $N$, then

$$
\begin{aligned}
\| f(y+u) & -\sum_{k=0}^{n} \frac{1}{k !} \cdot\left(P_{k}(y)\right) u^{k} \| \\
& \leqslant \text { l.u.b. }\left\|\frac{1}{0<\tau<1}\left\{\left(P_{n}(y+\tau u)\right) u^{n}-\left(P_{n}(y) u^{n}\right)\right\}\right\| .
\end{aligned}
$$

The special case of Theorem 3.1, when $n=1$, is proved by McLeod [11]. Dieudonné $[5$, p. 155] gives this result in less general form.

THEOREM 3.2. Let $f: A \rightarrow F$ and $f$ have a weak $n$-Taylor series expansion about every point of some neighbourhood $N$ of a point $x \in A$. If $y$ and $u$ are vectors such that the line segment $\{y+t u: 0 \leqslant t \leqslant 1\}$ is in $N$ and $P_{n}$ is continuous at $x$. Then for $0 \leqslant k \leqslant n, P_{k}$ has an expansion about $y$ of the form:

$$
P_{k}(y+s u)=\sum_{j=k}^{n}(1 /(j-k) !) \cdot\left(P_{j}(y)\right) u^{j-k}+r_{k}(s, u),
$$

where the remainder $r_{k}(s, u)$ has the property that for every $\varepsilon>0$, there exist a $\delta>0$ such that whenever $\|y-x\|<\delta / 2,|s|<\delta / 4$ and $\|u\| \leqslant 1$, then $\left\|r_{k}(s, u)\right\|<\varepsilon \cdot|s|^{n-k}$.

In particular, $P_{k}$ has a strong $(n-k)$-Taylor series expansion about $x$.

Proof. For any scalar $s$ and vector $y, u$, and $v$ we define a polynomial

$$
\begin{aligned}
(P(y, u, v, s)) t= & \sum_{k=0}^{n}(1 / k !) \cdot\left(P_{k}(y+s u)\right)(s t v)^{k} \\
& -\sum_{k=0}^{n}(1 / k !) \cdot\left(P_{k}(y)\right)(s u+s t v)^{k} .
\end{aligned}
$$

Both sums on the right are the approximations to $f(y+s u+s t v)$ corresponding to the expansions about $y+s u$ and about $y$.

Using the corollary to Theorem 3.1, 
$\|(P(y, u, v, s)) t\|$

$$
\begin{aligned}
& <\underset{0<\tau<1}{\operatorname{l.u.b} .}\left\|\frac{1}{n !}\left\{P_{n}(y+s u+\tau s t v)(s t v)^{n}-\left(P_{n}(y+s u)\right)(s t v)^{n}\right\}\right\| \\
& +\underset{0<\tau<1}{1 . u . b .}\left\|\frac{1}{n !}\left\{P_{n}(y+\tau(s u+s t v))(s u+s t v)^{n}-\left(P_{n}(y)\right)(s u+s t v)^{n}\right\}\right\| .
\end{aligned}
$$

We conclude that for a given $\varepsilon_{1}>0$ there is a $\delta>0$ such that whenever $\|y-x\|<\delta / 2$ and $|s|<\delta / 4$, then $\|P(y, u, v, s) t\|<\varepsilon_{1} \cdot|s|^{n}$, uniformly for $\|u\|<1,\|v\|<1$ and $0<t<1$, because of continuity of $P_{n}$ at $x$. Then a similar conclusion is true for the coefficients of $t^{k}, 0<k \leqslant n$, which is

$$
\left(s^{k} / k !\right) \cdot\left(P_{k}(y+s u)\right) v^{k}-\sum_{j=k}^{n} \frac{1}{(k !)(n-k) !} \cdot\left\{s^{j} \cdot\left(P_{j} y\right)\left(u^{j-k} v^{k}\right)\right\}
$$

so that using (2.7),

$$
\left\|\left(P_{k}(y+x u)-\sum_{j=k}^{n}(1 /(j-k) !)\right) \cdot\left(P_{j}(y)(s u)^{j-k}\right)\right\|<\varepsilon_{1} \cdot A \cdot|s|^{n-k},
$$

for $k=0,1,2, \ldots, n$, whenever $\|(y-k)\|<\delta / 2$ and $|s|<\delta / 4$, where $A$ is a constant depending only on $n$.

THEOREM 3.3. Let $f: A \rightarrow F$ and $f$ have a weak $n$-Taylor series expansion about every point of some neighbourhood $N$ of a point $x \in A$. If $y$ and $u$ are vectors such that the line segment $\{y+t u: 0<t<1\}$ is in $N$ and $P_{n}$ has a strong $n$-Taylor series expansion about $x$ of the form

$$
P_{n}(x+u)=\sum_{i=0}^{m}(1 / i !) \cdot \alpha_{i} u^{i}+r_{n}(u)
$$

then for $0<k \leqslant n, P_{k}$ has a strong $(n+m-k)$-Taylor series expansion about $x$ of the form:

$$
\begin{aligned}
P_{k}(x+u)= & \sum_{j=k}^{n-1} \frac{1}{(j-k) !} \cdot\left(P_{j}(x)\right) u^{j-k} \\
& +\sum_{i=0}^{m} \frac{1}{(i+n-k) !} \cdot \alpha_{i} u^{i+n-k}+r_{k}(u)
\end{aligned}
$$

where $r_{k} /\|u\|^{m+n-k} \rightarrow 0$ as $u \rightarrow 0$.

ProOF. Let $g(x+u)=f(x+u)-\sum_{j=0}^{m}(1 /(j+n) !) \cdot \alpha_{j}(u)^{n+j}$. Then $g(y$ $+u)=f(y+u)-\sum_{j=0}^{m}(1 /(j+n) !) \cdot \alpha_{j}(u+y-x)^{n+j}$, so that $g$ has a weak $n$-Taylor series expansion about $y$ :

$$
g(y+t u)=\sum_{k=0}^{n} t^{k} \cdot(1 / k !) \cdot\left(Q_{k}(y)\right) u^{k}+r(t, u),
$$

where

$$
Q_{k}(y)=P_{k}(y)-\sum_{j=0}^{m}(1 /(j+n-k) !) \cdot \alpha_{j}(y-x)^{n+j-k}
$$


and $\lim _{t \rightarrow 0} r(t, u) / t^{n}=0$ by the given hypothesis. Then

$$
\lim _{y \rightarrow x}\left(Q_{n}(y)\right) /\|(y-x)\|^{m}=0
$$

by the hypothesis of the theorem. If the method of proof of Theorem 3.2 is used for the expansion of $g$ about $x+s u$ and $x$, then by Theorem 3.1 and (3.4),

$$
\frac{\lim _{s \rightarrow 0}\left[\left(Q_{k}(x+s u)\right) v^{k}-\sum_{j=k}^{n}(1 /(j-k) !) \cdot s^{j-k} \cdot\left(Q_{j}(x)\right)\left(u^{j-k} v^{k}\right)\right]}{s^{m+n-k}}=0,
$$

$k=0,1,2,3, \ldots, n$, uniformly for $\|u\|<1,\|v\|<1$. Therefore

$$
\frac{\lim _{s \rightarrow 0}\left[Q_{k}(x+s u)-\sum_{j=k}^{n}(1 /(j-k) !) \cdot s^{j-k}\left(Q_{j}(x)\right) u^{j-k}\right]}{s^{m+n-k}}=0,
$$

uniformly for $\|u\| \leqslant 1$.

In terms of $f$, this implies

$$
\begin{aligned}
P_{k}(x+s u)= & \sum_{j=k}^{n-1}(1 /(j-k) !)\left(P_{j}(x)(s u)^{j-k}\right) \\
& +\sum_{j=0}^{m}(1 /(n+j-k) !) \alpha_{j}(s u)^{n+j-k}+r(s, u)
\end{aligned}
$$

where $r / s^{m+n-k} \rightarrow 0$ as $s \rightarrow 0$ uniformly for $\|u\|<1$, noting that

$$
\begin{aligned}
\left(Q_{0}(x)\right), & \left(Q_{1}(x)\right), \ldots,\left(Q_{n-1}(x)\right) \\
& =\left(P_{0}(x)\right),\left(P_{1}(x)\right),\left(P_{2}(x)\right), \ldots,\left(P_{n-1}(x)\right)
\end{aligned}
$$

and that $\left(Q_{n}(x)\right)=0$.

Thus $P_{k}$ has a strong $(n+m-k)$ Taylor series expansion as asserted.

4. Converse of Taylor's theorem. The converse of Taylor's theorem is not true without further conditions. A converse to the W. H. Yong form which characterises $n$th order Fréchet differentiability at a point is given by M. Z. Nashed [12] who imposes conditions on the remainder $R_{n}(x, u)$ that it be $(n-1)$ times Fréchet differentiable with respect to $u$ in a deleted neighbourhood of 0 ) and that $\lim _{u \rightarrow 0}\left(R_{n}^{(j)}(u)\right) /\|u\|^{n-j}=0, j=0,1,2, \ldots, n-1$. The converse which characterises $n$-Fréchet differentiability on a region is given by Abraham and Robin [1] who impose conditions on the remainder, together with the continuity of all the coefficients of Taylor's expansion (see the theorem below).

Theorem (ABraham AND RoBin). $A$ function $f: A \rightarrow F$ has a continuous nth order Fréchet differential on $A$ (in the iterated sense) if and only if there exist mappings $P_{k}: A \rightarrow L_{k}(E, F), k=0,1,2, \ldots, n$, which are continuous and

$$
f(y+u)=\sum_{k=0}^{n} 1 /(k !) \cdot\left(P_{k}(y)\right) u^{k}+r(y, u),
$$


where $\lim _{(y, u) \rightarrow\left(y_{0}, 0\right)}\|r(y, u)\| /\|u\|^{n}=0$ for every $y_{0} \in A$.

This theorem was proved in one-dimensional case by Marcinkiewicz and Zygmund [10] and for mappings $R^{n} \rightarrow R$ by Glasser [6].

We obtain a converse of Taylor's theorem as a consequence of Theorem 3.2 which holds with very weak hypothesis namely a weak $n$-Taylor series expansion about every point of a neighbourhood of $x$ together with the continuity of $P_{n}: A \rightarrow L_{n}(E, F)$ at $x$ and boundedness of $P_{0}(x), P_{1}(x), \ldots, P_{n}(x)$ as multilinear functions (we do not assume that $\left(P_{k}(y)\right)$ are bounded $k<n$ and $y \neq x$; this is a conclusion).

THEOREM 4.1. Let $f: A \rightarrow F$ have a weak $n$-Taylor series expansion about every point of some neighbourhood $N$ of a point $x \in A$. Suppose that at one point $x,\left(P_{1}(x), \ldots,\left(P_{n}(x)\right)\right)$ are bounded and $P_{n}$ is continuous at $x$. Then for $1<k<n-1$ and $y$ near $x,\left(P_{k}(y)\right)$ is Fréchet differential of $P_{k-1}$ at $y$ and $P_{n}(x)$ is Fréchet differential of $P_{n-1}$ at $x$.

Proof. First for $y$ near $x,\left(P_{j}(y)\right)$ is a bounded multilinear function because

$$
P_{k}(y)=\sum_{j=k}^{n}(1 /(j-k) !) \cdot\left(P_{j}(x)\right)(y-x)^{j-k}+r(y),
$$

derived from (3.3). Then if we use (3.3) to estimate $P_{k}(y+s u)$, all but two terms may be transposed to the right to go with the remainder. In the case $k=n-1$, it is necessary to have the number in the description $r_{n-1}$ approach 0 , and the same proof is valid at $x$ only.

Remark. In fact a careful observation shows that $P_{n}(x)$ is a strong differential of $P_{n-1}$ at $x . P_{n-1}$ is uniformly continuous in a neighbourhood of $x$ and all differentials $P_{k}$ as described are strong differentials.

The author is highly indebted to Professor Ernest B. Leach for his able guidance during the preparation of this paper. The author is also thankful to the Department of Mathematics, Case Western Reserve University, Cleveland, Ohio, for providing financial support.

\section{REFERENCES}

1. R. Abraham and J. Robin, Transversal mappings and flows, Benjamin, New York, 1967. MR 39 \#2181.

2. S. Dayal, Local representation and higher differentiability of $n$-convex operators on Banach spaces (to appear).

3. L Local representation of functions on normed linear spaces, Ph.D Thesis, Case Western Reserve Univ., Cleveland, Ohio, 1972.

4. __ A converse of Taylor's theorem for functions on locally convex and topological linear spaces (to appear).

5. J. Dieudonne, Foundations of modern analysis, Academic Press, New York, 1960. MR 22 \#11074.

6. G. Glasser, Etude de quelques algèbres Tayloriennes, J. Analyse Math. 6 (1958), 1-124.

7. Francis B. Hilderbrand, Introduction to numerical analysis, McGraw-Hill, New York, 1956. MR 17, 788. 
8. J. Kopec and J. Musielak, On the estimation of the norm of the n-linear symmetric operators, Studia Math. 15 (1955), 29-30. MR 17, 512.

9. E. B. Leach, A note on inverse function theorems, Proc. Amer. Math. Soc. 12 (1961), 694-697. MR 23 \#A3442.

10. J. Marcinkiewicz and A. Zygmund, On the differentiability of functions and summability of trignometric series, Fund. Math. 26 (1936), 1-43.

11. R. M. McLeod, Mean value theorems for vector valued functions, Proc. Edinburgh Math. Soc. (2) 14 (1964/65), 197-229. MR 32 \# 2522.

12. M. Z. Nashed, Differentiability and related properties of nonlinear operators: Some aspects of the role of differentials in nonlinear functional analysis, Nonlinear Functional Anal. and Appl. (Proc. Advanced Sem., Math. Res. Center, Univ. of Wisconsin, Madison, Wis., 1970), Academic Press, New York, 1971, pp. 103-309. MR 43 \#2580.

13. A. E. Taylor, Addition to the theory of polynomials in normed linear spaces, Tôhoku Math. J. 44 (1938), 302-318.

Department of Mathematics, Rohtak University, Rohtak (HaRyana), Indi

Current address: Department of Mathematics, University of Sulaimaniyah, Sulaimaniyah, Iraq 